\title{
Alternating regimes of motion in cell motility models
}

\author{
Nara Guisoni, ${ }^{1, *}$ Karina I. Mazzitello, ${ }^{2, \dagger}$ and Luis Diambra ${ }^{3, \ddagger}$ \\ ${ }^{1}$ Instituto de Investigaciones Fisicoquímicas Teóricas y Aplicadas (INIFTA), Universidad Nacional de La Plata - CONICET \\ ${ }^{2}$ Instituto de Investigaciones Científicas y Tecnológicas en Electrónica, \\ Universidad Nacional de Mar del Plata - CONICET \\ ${ }^{3}$ Centro Regional de Estudios Genómicos, \\ Universidad Nacional de La Plata - CONICET.
}

\begin{abstract}
Cellular movement is a complex dynamic process, resulting from the interaction of multiple elements at the intra and extra-cellular levels. This epiphenomenon presents a variety of behaviors, which can include normal and anomalous diffusion or collective migration. In some cases cells can get neighborhood information through chemical or mechanical cues. A unified understanding about how such information can influence the dynamics of cell movement is still lacking. In order to improve our comprehension of cell migration we consider a cellular Potts model where cells move actively in the direction of a driving field. The intensity of this driving field is constant, while its orientation can evolves according to two alternative dynamics based on the Ornstein-Uhlenbeck process. In the first case, the next orientation of the driving field depends on the previous direction of the field. In the second case, the direction update considers the mean orientation performed by the cell in previous steps. Thus, the latter update rule mimics the ability of cells to perceive the environment, avoiding obstacles and thus increasing the cellular displacement. Our results indicate that both dynamics introduce temporal and spatial correlations in cell velocity in a friction coefficient and cell density dependent manner. Furthermore, we observe alternating regimes in the mean square displacement, with normal and anomalous diffusion. The crossovers between superdiffusive and diffusive regimes, are strongly affected by both the driving field dynamics and cell-cell interactions. In this sense, when cell polarization update grants information about the previous cellular displacement decreases the duration of the diffusive regime, in particular for high density cultures.
\end{abstract}

\section{INTRODUCTION}

Cell motion plays a key role in many physiological ${ }^{53}$ processes including tissue morphogenesis, wound healing, ${ }^{54}$ immune and inflammatory response. It is known that the ${ }^{55}$ movement of cells is strongly influenced by cell-cell inter- ${ }^{56}$ actions, which grants a wide spectrum of behaviors. Cell ${ }^{57}$ motion can be categorized in terms of its external en- ${ }^{58}$ vironment, which can present directional asymmetry in ${ }^{59}$ response to a chemical stimulus, or be isotropic, without ${ }^{60}$ a preferred direction. In this sense, the single cell track- ${ }^{61}$ ing technique provides substantial evidence that in the ${ }^{62}$ absence of chemotactic cues, cells perform a persistent ${ }^{63}$ random walk, which has been modeled by the Ornstein- ${ }^{64}$ Uhlenbeck (OU) process [1]. In the case of directional ${ }^{65}$ asymmetry of the environment the cell is said to perform ${ }^{66}$ taxis and cell movement has been widely modeled using ${ }^{67}$ the Keller-Segel diffusion equation [2]. In addition to this ${ }^{68}$ categorization, cell motion can refer to the movement of 69 individual cells with or without neighbors $[3,4]$, or to $\mathrm{a}^{70}$ cell population acting as an aggregate [5-7].

Experimental results focused on individual movements ${ }^{72}$ share characteristics of Brownian particles [8, 9]: ex- ${ }^{73}$ ponential decay of the velocity autocorrelation function ${ }^{74}$ $(\mathrm{ACF})$ and linear growth with time of the mean square ${ }^{75}$ displacement (MSD), at large time scales [10]. These fea- ${ }^{76}$

\footnotetext{
*naraguisoni@gmail.com

$\dagger$ kmazzite@gmail.com

¥ ldiambra@gmail.com
}

51 tures can be explained by the OU process, or equivalently, the conventional Klein-Kramers description [1, 11]. However, there exist cell motions without chemotaxis that do not follow the OU process, for example, movements of epithelial cells and aggregates of Hydra cells, reported as anomalous diffusion processes [5]. Also human fibroblasts and keratinocytes move in a manner that contradict the OU process [5]. Similarly, Takagi et al. have reported different cell movement behaviors with anomalous diffusion for Dictyostelium cells in different physiological conditions [12]. These experiments fit well with a generalized Langevin model that includes a memory kernel for cell velocity [13]. Furthermore, long-term analysis of MDCK cells has revealed a superdiffusive behavior, in absence of external cues [4, 14]. These findings show that cell movement contains a more complex dynamics than the persistent random walk, which can be explained by the fractional Klein-Kramers equation [4]. The latter can be considered as a phenomenological approach, able to describe anomalous diffusion in terms of very general physical mechanisms. However, it has limitations to indicate which biological ingredients lead to an anomalous behavior. Thus, alternative modeling that allows to get biological insight by testing different hypotheses becomes really interesting.

Recently, we have introduced a reorientation model based on the cellular Potts framework [15]. In this model, cell movement due to a driving field, which direction changes following a discrete version of the OU process, was considered. In contrast, previous models have applied the OU process on the velocity vector, leading to white-noise fluctuations on the direction angle 
$[8,9,16,17]$. It is known that when the orientation angle fluctuates without correlations (i.e., in the absence of the friction term) the system exhibits Brownian motion $[16,18]$. However, when the friction term is present, we found that high density cultures exhibited a doubleexponential for the velocity ACF, in contrast to an exponential characterizing the Brownian motion of low density cultures. For both densities the MSD behaves as a persistent random walk model for the time scale studied [15]. These results suggest that the complex behavior of ${ }_{137}^{130}$ cell motion can be consequence of the intrinsic feature of ${ }_{138}^{137}$ the movement, but also of cell-cell interactions.

In this paper, we are particularly interested in under-10 standing the interplay between the neighborhood infor-141 mation gathered by the cell in previous displacement and $_{142}$ cell motility over large time scales. To address this ques-143 tion, the cellular Potts model (CPM) introduced in $[15]_{144}$ with two types of dynamics for the orientation angle of $f_{145}$ cell displacements are compared. Firstly, we consider ${ }_{146}$ a "naive" implementation where the new orientation is ${ }_{147}$ related to the field direction operating in the previous ${ }_{148}$ step, regardless the cellular direction of displacement. $\operatorname{In}_{149}$ the second case, the update direction depends on the ${ }_{150}$ mean orientation performed by the cell in the previous ${ }_{151}$ steps. This implementation of the field direction update ${ }_{152}$ grants a sort of feedback mechanism, because at each ${ }_{153}$ time step the angle of the driving field is influenced by ${ }_{154}$ the recent cell history, taking into account interactions ${ }_{155}$ between cells blocking and deviating from their original orientations. We compare both actualization models analyzing the MSD, the temporal and spatial correlations of cell velocity and the average distance traveled by a cell during the time interval that the driving field is operating, at low and high density cultures.

\section{THE MODEL}

The CPM is a modified Potts model which includes different terms of energy that become it able to repro-164 duce some biophysical properties of cells, such as defor- -165 mations of cell membrane, adhesion and motility in $\mathrm{an}_{166}$ excluded volume manner. In the model, at each site of $f_{167}$ the lattice a spin $\sigma_{i}=1, \ldots, Q$ is assigned, and cells are ${ }_{168}$ represented by domains with the same spin, thereby $\mathrm{if}_{169}$ $\sigma_{i}=M$, with $1 \leq M \leq Q$, it belongs to the cell labeled ${ }_{170}$ as $M$. The dynamics of the model are governed by the ${ }_{171}$ Hamiltonian, or energy function, which guides the cell $1_{172}$ behavior by distinguishing the low energy configurations ${ }_{173}$ (or favorable) from the high energy ones. The Hamilto- ${ }_{174}$ nian is constituted by a term corresponding to the $\operatorname{sum}_{175}$ of all surface energies, responsible for cell-cell adhesion ${ }_{176}$ properties. However, to keep the cells without that they ${ }_{177}$ be broken or disappear, additional terms in the Hamil- ${ }_{178}$ tonian are needed. Thus, the energy function considered here is given by

$$
\begin{array}{r}
H_{0}=\sum_{i, j \text { neighbors }} J_{\sigma_{i} \sigma_{j}}\left(1-\delta_{\sigma_{i} \sigma_{j}}\right)+ \\
+\sum_{M=1}^{Q} \kappa\left(V_{M}-V_{0}\right)^{2}+\sum_{M=1}^{Q} \Gamma\left(L_{M}-L_{0}\right)^{2}
\end{array}
$$

where $\delta_{\sigma_{i} \sigma_{j}}$ is the Kronecker delta and the first sum is over all neighboring site pairs, representing the boundary energy of the interacting cells. The second and third terms in Eq. (1) correspond to the energy costs for cells to deviate from the preferred volume $V_{0}$ and perimeter $L_{0}$, respectively. The presence of a medium, which interacts with the cells, is also considered. In this way, the medium has spin variable $\sigma_{i}=0$, with no target area or perimeter. The adhesion constant between different cells is denoted by $J_{\text {cell-cell }}$ whereas between cells and medium is $J_{\text {cell-medium }}$. Further, to consider cell motility preferentially along the direction of a driving field $\vec{F}$, an additional term should be added to the Hamiltonian Eq. (1) $[15,19]$, as we will see below.

The system evolves using Monte Carlo dynamics. In order to obtain a new configuration a lattice site is randomly chosen and if it belongs to the boundary of the cell, this site copies the spin value of one of its neighboring cells as a trial. The variation of energy in a proposed trial configuration is given by

$$
\Delta H=\Delta H_{0}+\sum_{M=1}^{Q} \vec{F}_{M} \cdot \Delta \vec{r}_{M},
$$

where $\Delta H_{0}$ is the change of energy related to Eq. (1), $\vec{r}_{M}$ denotes the displacement of the center of cell $M$ and $\vec{F}_{M}$ is the driving field acting on cell $M$. The acceptance, or not, of a new configuration is given by the Metropolis prescription: the trial is accepted with probability 1 if it decreases the value of energy, $\Delta H \leq 0$, or with the Boltzmann factor $e^{\left(-\Delta H / k_{B} T\right)}$ if it increases the energy $(\Delta H>0)$, where $k_{B} T$ parameterizes the intrinsic membrane motility. The unit of time, a Monte Carlo step (MCS), is defined as $N$ trials of movement, being $N$ the number of spins in the lattice.

The driving field is characterized by a direction, denoted by $\Theta$, and an intensity $F$. We consider that the intensity $F$ is constant over time and the same for all cells. However, the direction of the driving field operating over cell $M, \Theta_{M}$, is actualized according to OU process, $d \Theta_{M}(t)=-\lambda \Theta_{M}(t) d t+\sigma d W(t)$, where $\lambda$ is the friction coefficient $(0 \leq \lambda<1), \sigma$ determines the magnitude of the fluctuations and $d W(t)$ denotes the Wiener process. For our Monte Carlo simulations, it is necessary to use a discrete version of this stochastic differential equation, which can be identified with a first-order autoregressive process, as follow:

$$
\Theta_{M}(n)=(1-\lambda) \Theta_{M}(n-1)+\sigma \epsilon(n), \quad n=1,2, \ldots(3)
$$


where $\epsilon(n)$ is a white noise with zero mean and unit 232 variance $\left(\sigma_{\epsilon}^{2}=1\right)$ and $n$ is the discrete time. $\lambda$ and $\sigma_{233}$ were defined previously.

Note that it is not mandatory that the cell displace-235 ment has the same direction of the associated driving 236 field, due to both cell-cell interactions and stochastic fluc-237 tuations. Thus, the angle of the driving field $\Theta_{M}$ is not 238 necessarily equal to the direction of the cell movement,239 which will be denoted by $\alpha_{M}$. If the previous direction of the cell displacement is considered in the actualization of the driving angle we have a positive feedback ${ }^{241}$ which mimics the situation in which the cell produces its ${ }^{242}$ own chemotactic signal. This aspect was taken into ac- ${ }^{243}$ count previously by other authors in Potts like models ${ }^{244}$ $[6,20,21]$. In that way, Kabla considers that the motile ${ }^{245}$ force is oriented along the mean velocity of the cell over ${ }^{246}$ its past time steps, without friction nor noise [21]. For $^{247}$ Szabó et al. [6] the change in cell polarization is pro- $^{248}$ portional to a spontaneous decay respect to its previous ${ }^{249}$ value and a reinforcement from cell displacement direc- ${ }^{250}$ tion during the time step considered. In order to take ${ }^{251}$ into account this feedback loop, an alternative way to ${ }^{252}$ update the angle of the driving field $\Theta_{M}$ is:

$$
\Theta_{M}(n)=(1-\lambda) \overline{\alpha_{M}}(n-1)+\sigma \epsilon(n),
$$
where $\overline{\alpha_{M}}(n-1)$ is the mean displacement angle over $^{256}$ the last $\tau$ MCS, and the other parameters are the same 258 as in Eq. (3). Thus, according to Eq. (4) the angle of the 259 driving force depends on the earlier displacements of the 260 cell. Differently from previous formulations $[6,20,21]_{, 261}$ our proposal for the feedback loop takes into account ${ }_{262}$ fluctuations. Besides, an advantage of Eq. (4) is the $\mathrm{e}_{263}$ possibility of a direct comparison with Eq. (3): the only ${ }_{264}$ difference between them is the dependence on the mean $_{265}$ cell polarity $\overline{\alpha_{M}}$ instead of on the previous direction of $\mathrm{f}_{266}$ the driving field $\theta_{M}$.

For both actualization procedures, the initial direction ${ }_{268}$ of the cell $M, \Theta_{M}(0)$, is chosen randomly between $[0,2 \pi]{ }_{269}$ $\Theta_{M}$ evolves independently of the field operating in other ${ }_{270}$ cells. The updating time in Eqs. 3 and $4, n$, is different ${ }_{271}$ from the time of actualization of cell configurations. $\operatorname{In}_{272}$ particular, at each time step the direction of the driving 273 field for each cell $M$ changes with probability $1 / \tau$ accord- ${ }_{274}$ ing to Eqs. 3 and 4. Thus, the change in the directions ${ }_{275}$ $\Theta_{M}$ and $\alpha_{M}$ occurs at a mean time $\tau$ independently of $\mathrm{f}_{276}$ the direction of other cells.

\section{RESULTS}

For all simulations in this paper, we used the following 282 fixed parameter values $J_{\text {cell-cell }}=0.1, J_{\text {cell-medium }}=283$ $0.01, \Gamma=0.2, \kappa=1, \sigma=\pi / 3, F=10, T=2 \operatorname{and}_{284}$ $\tau=10$. The density $\rho$ is defined as the ratio between 285 the area occupied by the cells and the medium. In this 286 way, we calculate the number of spins with $\sigma_{i} \neq 0$ re-287 lated to the total number of spins, since the medium is 288 identified by $\sigma_{i}=0$. Low and high density simulations 289 correspond to $\rho=0.2$ and $\rho=0.9$, respectively. Also, we considered along the paper three different values of friction coefficient $\lambda=0.01,0.05$ and 0.10 . We used periodic boundary conditions and a square lattice of size $1024 \times 1024$ sites. More details about initial conditions and thermalization can be found in [15].

In order to characterize the movement of a cell population we calculate the mean-squared displacement (MSD) as $\operatorname{MSD}(\mathrm{t})=\left\langle\left(\vec{r}_{\mathrm{M}}(\mathrm{t})-\vec{r}_{\mathrm{M}}(0)\right)^{2}\right\rangle$, where the average is taken over all cells of the simulation between a common starting point at $t=0$ and the actual positions at time $t$. According to Fig. 1, the MSD presents two or three regimes in the time scale considered, depending on the value of $\lambda$ and on the updating rule used. For $\lambda=0.01$ and the OU actualization (Eq. 3), we can see that at short times the MSD is almost ballistic and after that it resembles a random walk, regardless the density. For the other situations, the MSD has three regimes: it is almost ballistic at short times, diffusive at intermediate time scale and superdiffusive at long times. When the direction of the driving field is actualized by using OU with feedback (Eq. 4), the crossover between the random walk behavior and the superdiffusive regime at long times occurs previously for $\lambda=0.10$ than for 0.01 . On the other hand, when the OU actualization is used, this crossover is present for $\lambda=0.10$ but not for 0.01 . Consequently, the duration of the diffusive period is shorter when the friction coefficient is higher (same actualization model and different values of $\lambda$ ) and when the feedback is present (same value of $\lambda$ and different actualization models). In order to understand these results, let us discuss the meaning of the different regimes observed for the MSD along the different time scales. For all cases, the almost ballistic short-time behavior is related to the persistence time of the driving force, $\tau$. In fact, in a previous paper [15] we shown that the temporal behavior of the MSD scales with $\tau$, for the OU actualization. The diffusive behavior of the MSD, also present for all cases shown in Fig. 1, is the result of the fluctuations in the direction of the driving field, since Eqs. 3 and 4 are stochastic equations. However, the actualization of $\Theta$ is not completely random since the friction coefficient $\lambda$ introduces correlations in the successive directions of the cells displacements, whose influence is observed at long times. This effect is particularly evident when using Eq. 4 , since the presence of feedback rises the correlations, and therefore favors the anomalous diffusive behavior.

Also, from Fig. 1, the MSD obtained from the angle updating rule with feedback is greater or equal than that found with the OU actualization. This result suggests that feedback helps cells avoid collisions with another cells, making the movement more effective. Finally, we discuss the effect of density on the MSD, starting with the model of OU actualization. Low density configurations have higher or equal MSD than the obtained for high density, for both values of $\lambda$ (see black lines in Fig. 1). In fact, a lower MSD for high density cultures is expected, since crowded cell cultures usually disturb the movement 

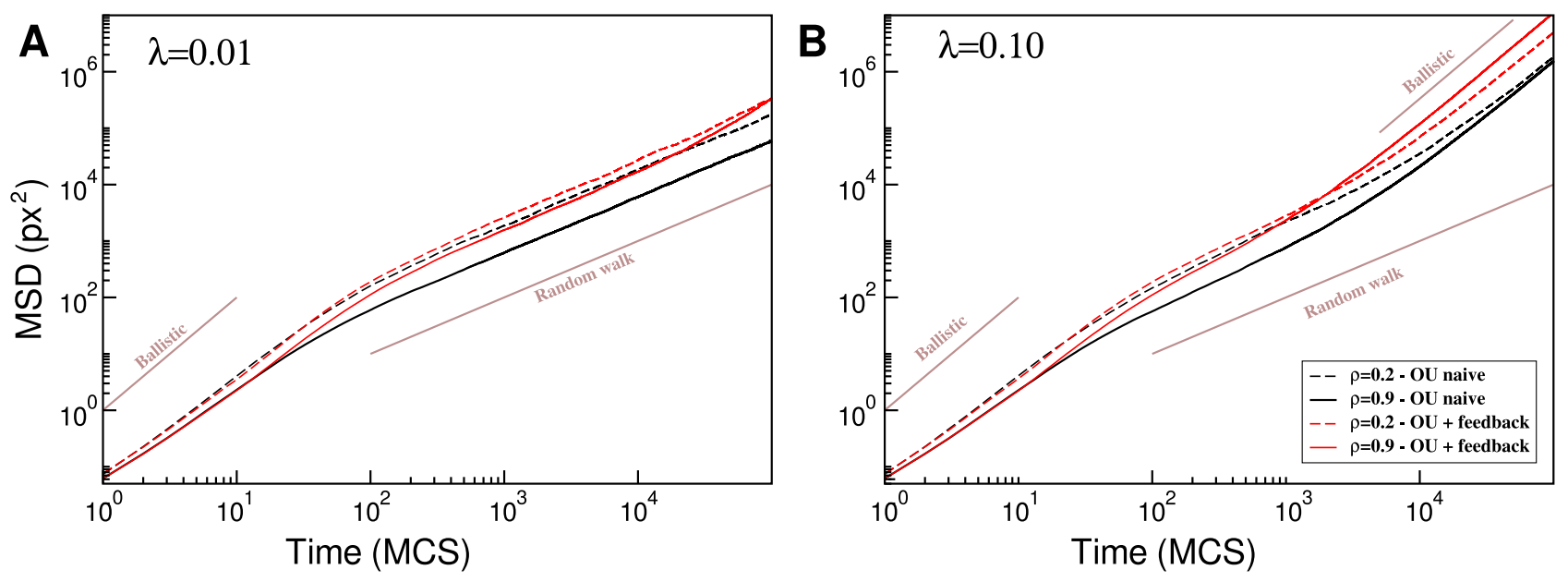

FIG. 1. Log-log plot of the mean-squared displacement, msd, vs. time, $t$. Direction of the driving field actualized according to Ornstein-Uhlenbeck (OU naive) and Ornstein-Uhlenbeck with feedback (OU + feedback), $\lambda=0.01(\mathrm{~A})$ and $\lambda=0.10$ (B), and $\rho=0.2,0.9$ (dashed and continuous line, respectively). Lines with slope equal 2 (Ballistic) and 1 (Random walk) are shown for the sake of comparison.

of cells. The same behavior related with density can be 325 observed at short and intermediate times when the feed-326 back updating rule is used. However, at long times, the 327 high density culture presents higher MSD than the low 22 density one, for $\lambda=0.10$ and feedback update. This in-329 version in the MSD suggests that the feedback gives rise $з о 0$ to a spatially coordinated movement of the cells in high ${ }_{331}$ density simulations.

The temporal behavior of MSD scales with time a $\operatorname{MSD}(\mathrm{t}) \sim \mathrm{t}^{\beta(\mathrm{t})}$, where the exponent $\beta$ characterizes the $\mathrm{e}_{335}$ different regimes observed. In that way, a ballistic be- ${ }_{336}$ haviour is associated with $\beta=2$, whereas normal diffu- ${ }_{337}$ sion presents $\beta=1$. The logarithmic derivative of the $\mathrm{e}_{338}$ MSD allows the calculation of $\beta$ as $\beta(t)=\frac{d \ln \operatorname{MSD}(\mathrm{t})}{d \ln (t)} .339$ Similar measurements were used to study cell migration ${ }^{340}$ [4] and intracellular transport [22] from both experiments ${ }^{341}$ and models, and for a simple model that mimics the dif-342 fusion of a particle in an anisotropic amorphous material ${ }^{343}$ [23]. Fig. 2 shows the behavior of $\beta$ as obtained from the ${ }^{344}$ two angle updating rules, for low (dashed lines) and high ${ }^{345}$ (solid lines) densities and different values of $\lambda$. $\beta$ was was $^{346}$ computed using a time-sliding window, the size of which ${ }^{347}$ depends on $t$. At the short-time scale the exponent $\beta_{348}$ corresponds to anomalous diffusion. At this scale, we $\mathbf{w}_{49}$ can note that for the OU actualization, low density cul-350 tures present higher $\beta$ than high density. This aspect is ${ }_{351}$ less evident when the feedback mechanism is considered,352 since $\beta$ for low and high density cultures presents almost 353 the same value in the range $[30,100]$ MCS. Besides, at ${ }_{354}$ short-time scale it can be seen a slight increase in $\beta$ for 355 the update with feedback. These results indicate that 356 the feedback makes cell movement more efficient, partic-357 ularly for high density cultures, as discussed before. At 358 intermediate time scale, $\beta$ decreases and the MSD tends359 to exhibit a diffusive behavior. Particularly, for the $\mathrm{OU}_{360}$ actualization and low $\lambda$-values, the diffusive behavior is observed at intermediate and long-time scales. For the other conditions of Fig. 2, the transition between shorttime and long-time superdiffusive regimes is so tight that the exponent $\beta=1$ is almost not reached, but will be referred to as a diffusive regime. The duration of this diffusive regime decreases with $\lambda$ (for the same angle updating rule) and with the feedback (for the same value of $\lambda$ ), as pointed out with Fig. 1. Also, for the OU actualization and low $\lambda$-values, $\beta$ is independent of the density at intermediate and long-time scale. For the other conditions in the same time scales, the high density cultures present higher $\beta$ than low density ones. Besides, from the behavior of $\beta$ at long-time scale when the feedback is considered (Fig. 2-B), we can suppose that for a larger time scale (that is for $t \geq 10^{5}$ MCS) $\beta$ will continue to grow until it reaches $\beta=2$. In fact, we can expect the same behavior for Fig. 2-A, since for both actualization rules there is correlation in the $\Theta$ update. As we have discussed previously, the correlation comes from the friction term being reinforced by the feedback. Cell-cell interactions also increase correlations at intermediate and long-time scales.

The velocity ACF is another important tool to characterize cell movement. It is defined as $C(t)=Z(t) / Z(0)$ where $Z(t)=\left\langle\vec{v}_{M}\left(t_{0}+t\right) \cdot \vec{v}_{M}\left(t_{0}\right)\right\rangle[24]$, and $\langle\ldots\rangle$ indicates the average over all cells and over $t_{0}$. The cell velocities are defined as $\vec{v}_{M}(t)=\Delta \vec{r}_{M}(t) / \Delta t$, where $\Delta \vec{r}_{M}(t)=\left(\vec{r}_{M}(t+\Delta t)-\vec{r}_{M}(t)\right)$, and $\Delta t=1 \mathrm{MCS}$. Fig. 3 shows that the velocity ACF obtained for the angle updating rule with feedback (Eq. 4) is always higher than the one obtained for the naive angle update (Eq. 3), regardless the values of $\lambda$ or $\rho$. In fact, if cells are more successful in avoiding collisions with another cells we expect a higher ACF. In Fig. 3, for the OU actualization, high density cultures have smaller ACF than low den- 

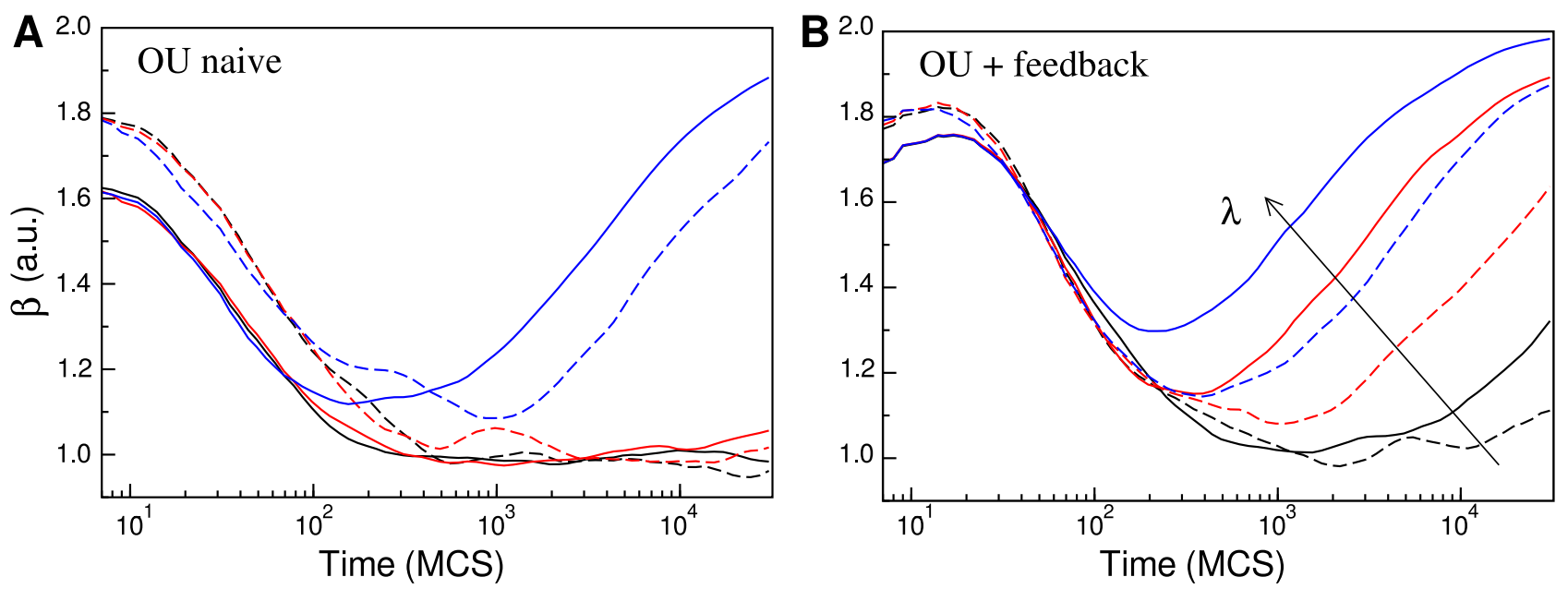

FIG. 2. Logarithmic derivative of the MSD, $\beta(t)$, as defined in the text, calculated from data shown in Fig. 1 and additional data. Direction of the driving field actualized according to OU naive (A) and OU with feedback (B) and different values of $\lambda=0.01,0.05,0.10$ (dark, red and blue, respectively) and $\rho=0.2,0.9$ (dashed and continuous line, respectively). We used a time-sliding window, the size of which depends on time. The interval between successive measurements is equal to $\left[1.775^{k}, 1.775^{(k+4)}\right]$ and $k=1,1.2,1.4, \ldots, 16$, since the MSD data was considered until $t=10^{5}$ MCS.
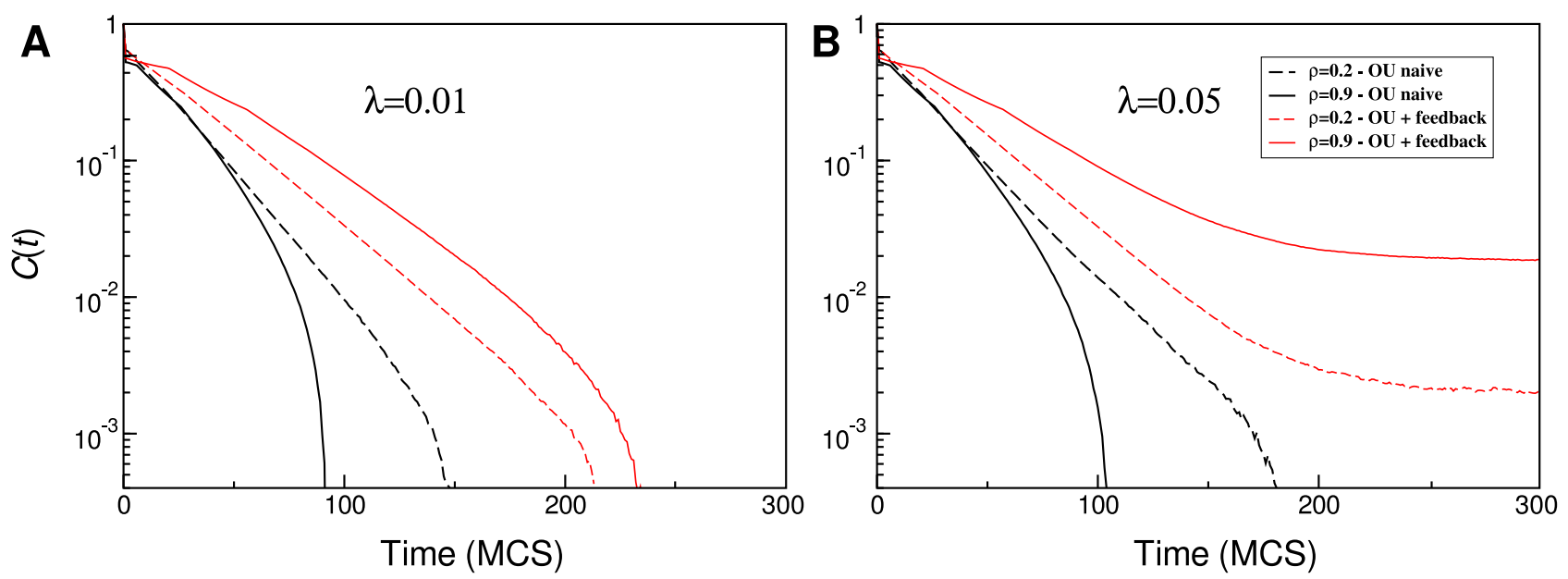

FIG. 3. Semi-log plot of the velocity ACF function, $C(t)$, vs. time, $t$. Direction of the driving field actualized according to OU naive and OU with feedback, for different densities, $\rho=0.2,0.9$ (dashed and continuous line, respectively), and friction coefficients, $\lambda=0.01,(\mathrm{~A})$ and $\lambda=0.05(\mathrm{~B})$. $C(t)$ was averaged over all cells in the simulations for $6 \times 10^{4}$ MCS and over 1 $(\rho=0.9)$ and 3 samples $(\rho=0.2)$.

sity cultures, for both $\lambda=0.01$ and 0.05 . This result 374 can be understood by the fact that a crowded neighbor-375 hood usually disrupts the movement of the cell. How-376 ever, when the feedback update is considered, this rela-377 tion is inverted: high density cultures have a higher $\mathrm{ACF}_{378}$ than low density cultures, independent of the value of $f_{379}$ $\lambda$. Therefore, we can conclude that the feedback makes $s_{30}$ cell movement more efficient mostly for high density cul-381 tures. Actually, when the update with feedback is used ${ }_{382}$ in high density cultures there is a competence between ${ }_{383}$ two effects: on the one hand a crowded environment hin-384 ders cell movement, and on the other hand, the feedback ${ }_{385}$ promotes it. But the OU update has only the first ef- ${ }_{386}$ fect. Because of that, the difference in the velocity $\mathrm{ACF}$ between the two update rules is more evident for high density cultures.

Also, Fig. 3 shows that the ACF is greater for $\lambda=0.05$ than for $\lambda=0.01$, regardless the density or the update model. This result indicates that the friction coefficient enhances the correlation in cell movement, as discussed before. Finally, for the OU with feedback update and $\lambda=0.05$ the ACF presents a very slow decrease at long times, consistent with an algebraic decay, independent of the density. For the other parameters considered in Fig. 3 the ACF goes to zero before $t=250$ MCS.

The spatial correlations of the cell velocities are also 

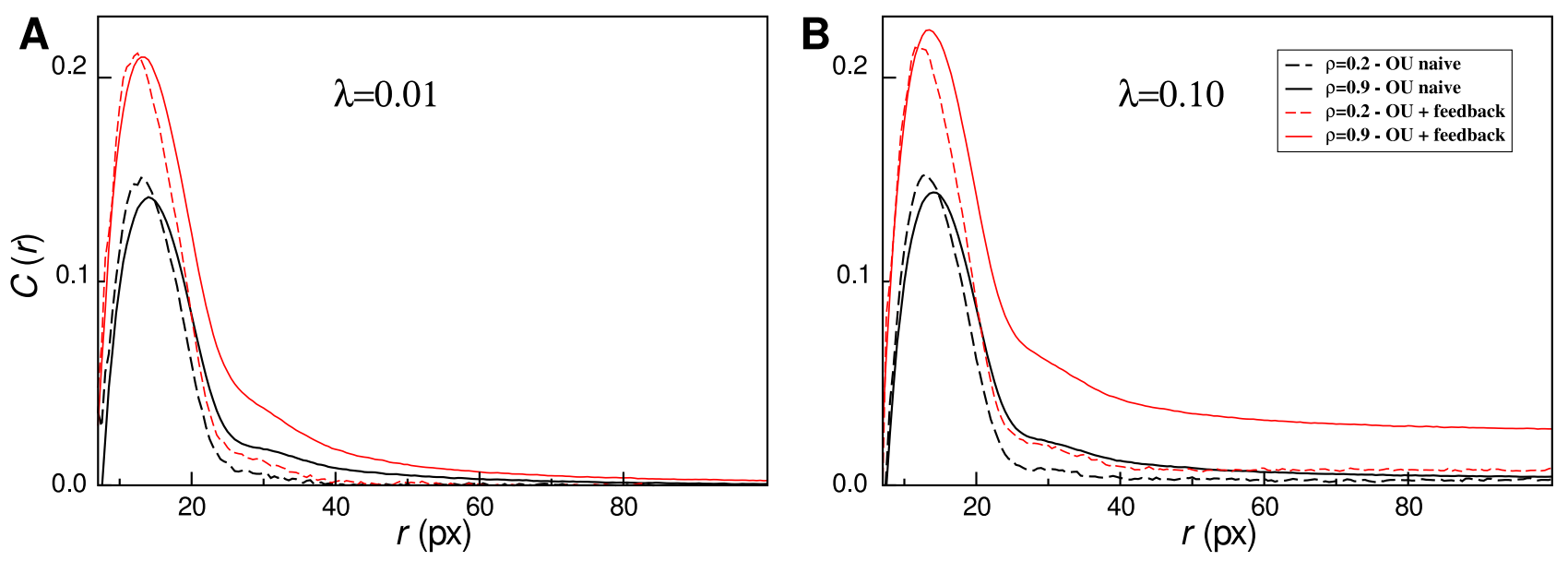

FIG. 4. Spatial correlation function of the velocities, $C(r)$, as a function of the distance $r$ between cell pairs. Direction of the driving field actualized according to OU naive and OU with feedback, for different densities, $\rho=0.2,0.9$ (dashed and continuous line, respectively), and friction coefficients, $\lambda=0.01,(\mathrm{~A})$ and $\lambda=0.10$ (B). Data were obtained by averaging over $10^{5}$ MCS.

used to study cell movement. It is defined as $C(r)={ }_{420}$ $\left\langle\vec{v}_{M} \cdot \vec{v}_{M^{\prime}} /\left(\left|\vec{v}_{M}\right|\left|\vec{v}_{M^{\prime}}\right|\right)\right\rangle$, with $r=\left|\vec{r}_{M}-\vec{r}_{M^{\prime}}\right|$ the 421 distance between mass center of cells $M$ and $M^{\prime}$. When ${ }_{422}$ comparing the two angle-update rules, we can see from ${ }_{423}$ Fig. 4 that $C(r)$ is always higher when the update rule 424 with feedback is used, as also observed for the $\mathrm{ACF}_{425}$ (Fig. 3). As discussed in a previous work [15], the peak 426 close to the typical diameter of the cell ( $\sim 16$ pixels $)$ is 427 related to anti-correlated velocities of cells that travel $l_{428}$ in opposite directions. At intermediate and long dis-429 tances $(r \gtrsim$ cell size) cells in high density configura-430 tions are more correlated, for both actualization rules431 and $\lambda$-values. Besides, $C(r)$ in high density simulations432 approaches zero very slowly, as $r$ increases, in particular 433 for $\lambda=0.10$ and when using the feedback update rule 434 (Fig. 4-B). These results indicate that cell-cell contact $t_{435}$ induces long-range spatial-correlation of cell velocity and 436 that this effect is enhanced by both the feedback and the ${ }_{437}$ friction coefficient. Also, the difference in $C(r)$ for low 438 and high density cultures is much higher in the case of up-439 date with feedback. Therefore, the effect of the feedback 440 raising spatial correlations is greater for the crowded cul-441 tures, as observed for the temporal correlations in Fig. 3.442 These results could indicate a coordinated movement of cells in high density cultures as a consequence of the feedback mechanism.

In order to get insight about the effect of the feedback ${ }^{43}$ introduced by the rule Eq. 4 on the cell movement, we define the average distance $\bar{D}$ traveled by a cell during ${ }^{444}$ the time interval that the driving field is operating in $\mathrm{a}^{445}$ given direction. Mathematically, it is defined as

$$
\bar{D}=\frac{1}{Q} \sum_{M=1}^{Q}\left\langle\left|\Delta \vec{r}_{M}\left(t_{f}-t_{i}\right)\right|\right\rangle_{I_{t}},
$$

where $\Delta \vec{r}_{M}\left(t_{f}-t_{i}\right)$ is the cell displacement from the $e_{451}$ time the driving field starts operating, $t_{i}$, until the time ${ }_{452}$ of the next direction change, $t_{f}$ (with $\left.t_{f}-t_{i} \sim \tau\right),\langle\ldots\rangle_{I_{t}}$ indicates the average over all time intervals $I_{t}=\left[t_{i}, t_{f}\right]$, and the sum runs over all cells on the substrate. This magnitude gives information about the cell movement only in the short-time scale $(\sim \tau$, i.e., during the first almost ballistic regime), for that reason it is nearly independent on $\lambda$-values, as we can see in Fig. 5 . Further, it is expected that cells can move much more in low density cultures than in a crowded media, which is also evident in Fig. 4 , where $\bar{D}$ is much higher for $\rho=0.2$ than for $\rho=0.9$. Fig. 5 also establishes a comparison of the magnitude $\bar{D}$ for the two angles-updating rules specified by Eqs. 3 and 4 . In all cases, the average distance $\bar{D}$ reached by the cells when the OU with feedback is acting is greater than when the OU update is applied. In particular, we observe that in the case of a crowded media there is a remarkable increment (of about $20 \%$ ) of $\bar{D}$ when the update with feedback is applied respect to the OU actualization. For the low-density case the increase is only about $3 \%$. These results suggest that the OU with feedback update promotes or increases cell displacement and that this effect is more noticeable in crowded environments.

\section{DISCUSSIONS AND CONCLUSION}

Much of the work about cell motility is based on the study of the time behavior of the second moment, the MSD. Thus, a system is considered to exhibit Brownian motion when the MSD increases linearly in time, otherwise it is considered to present anomalous diffusion. However, we usually want to know more about the cell trajectories than simply the second moment $[3,4]$. Other features of interest are the distribution and correlations of cell velocity. So, if normal diffusion occurs, the veloc- 


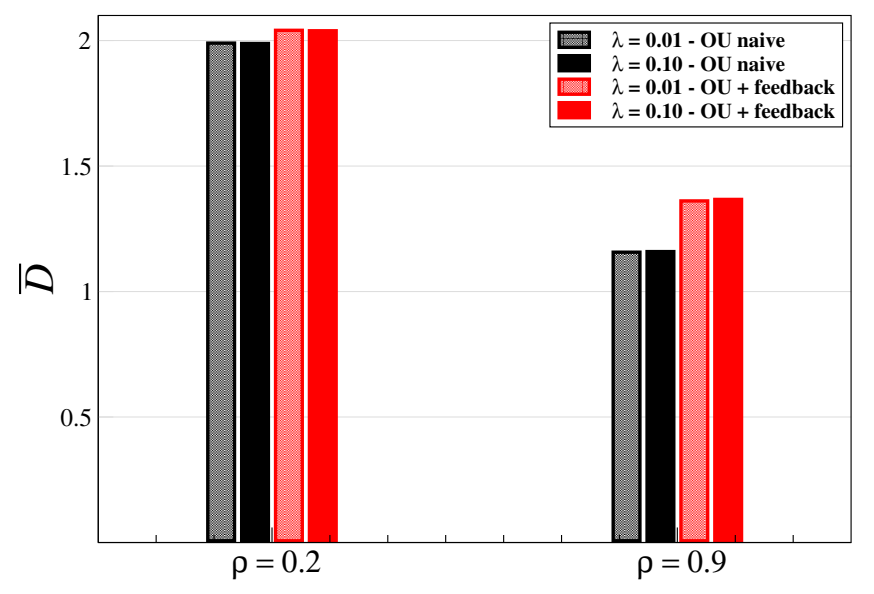

FIG. 5. Average distance travelled by a cell during the time interval that the driving field is operating, $\bar{D}$, for densities $\rho=0.2$ and $\rho=0.9$. Direction of the driving field actualized according to OU naive and OU with feedback (black and red, respectively) and friction coefficient $\lambda=0.01$, and $\lambda=0.10$. $\bar{D}$ was averaged over all cells in the simulations until $10^{5}$ MCS (the first 500 MCS were disregarded). Error bars are smaller than the thickness of the line.

ity correlation decreases to zero exponentially, or more 490 quickly, while anomalous diffusion could be associated ${ }_{491}$ with an algebraic decay of the velocity ACF. The $\mathrm{MSD}_{492}$ and the correlations can be theoretically derived only ${ }_{493}$ from simplified models $[13,16,17,22,25]$. This modeling 494 feature is particularly interesting to get insight about the 495 asymptotic behavior of the MSD. On the other hand, for 496 models which include biological ingredients like cell vol-497 ume and cell-cell interactions, as the CPM-based ones, it 498 is difficult to get analytical expressions. However, these 499 models can add valuable information about cell motion 500 $[6,15,21,26]$. For example, they are able to test feedback501 mechanisms from the neighbourhood at different spatial ${ }_{502}$ scale in an excluded volume schema [21, 26]. In addition,503 Kabla studies different cell dynamics for the collective504 movement resulting from the balance between adhesion505 and cell motile forces [21]. Here we study cell movement506 at long-time scales addressing the effect of cell-cell inter-507 actions and neighborhood information gathered by the 508 cell within the framework of a CPM-based model. We509 consider that cells move actively according to a driving ${ }_{510}$ field that has a constant intensity and whose orientation ${ }_{511}$ is governed by two alternative OU updating rules. The 512 proposed dynamics for the driving field provide both as13 persistent random walk feature, which include a sort of ${ }_{514}$ angle memory, as well as, a feedback mechanism able to515 mimics cell behavior with environment perception at lo-516 cal range.

We observed alternating superdiffusive-Brownian 518 regimes for the MSD in the temporal scale considered.519 The almost ballistic behavior at short time is related with 520 the persistence time $\tau$ of the driving field [15]. At inter-521 mediate time intervals the MSD becomes diffusive due to522 the random actualization of cell direction. A crossover 523 from a quadratic to a linear regime in the MSD has been524 previously reported in a model of self-propelled particles, 525 when the diffusive behavior arising from particle reorien-526 tation dominates the persistence process [17]. Besides, when the OU dynamics is applied to update particle velocities vector (instead to update the angle direction as was done here) the same crossover is observed in the MSD and the resulting asymptotic regime is Brownian $[1,16]$. In addition to this initial crossing of regimes, we found a second crossover between the diffusive behavior and a ballistic regime at large time scales. The second crossover occurs previously for large $\lambda$-values, since the stronger the friction term in the OU process, the higher the correlations. Furthermore, we show that this crossover also depends on the angle-update rule used, being favored when Eq. 4 is operating. This result is in agreement with the fact that temporal and spatial correlations are higher when the update rule with feedback is used. Also, our results suggest that the feedback update rule helps cells avoid collisions with another cells, making the movement more effective, and therefore contributing to supperdifusion. Finally, the transition to the long-term ballistic regime depends on cell-cell interactions and occurs previously in crowded cultures. Our findings can be related to previous results from a directed random walk model [27]. In this paper, the time of appearance of the asymptotic ballistic regime depends on an anisotropy parameter, which fixes the correlations in the displacement direction: at higher values of this parameter (or stronger anisotropy), there is more correlation and the ballistic regime appears early [27]. In the present work, we show that the appearance of the long time ballistic regime is favored by the friction term $\lambda$, by cell-cell interactions and also by the feedback updating rule. All these factors introduce correlations in cell movement, leading to a ballistic motion at long time scales. We also expect the crossover time between the diffusive and the ballistic regime be affected by $\sigma$, since greater values of $\sigma$ are related to a more stochastic cell movement and should correspond to a longer diffusive period. 
In conclusion, the two angle-updating rules used in539 interactions. this paper allow disentangling the effect of two sources of anomalies. Firstly, we observe that cellular motion governed by angle-updating rules with a no-null friction540 term, presents anomalous diffusion for long times, and more specifically a crossover from Brownian to ballistic $\mathrm{c}_{541}$ regimes. On the other hand, the temporal scale of the ${ }_{542}$ diffusive regime is shortened when the direction update s43 $_{54}$ rule includes a feedback mechanism. The characteristic scale where the crossover between these regimes is evident depends on the velocity correlations, which itself $f_{544}$ depends on the friction term, the feedback and cell-cell

\section{ACKNOWLEDGMENTS}

We acknowledge financial support from the Argentinian Science Agencies CONICET and ANPCyT, and from the UNLP (Universidad Nacional de La Plata).

\section{BIBLIOGRAPHY}

[1] George E Uhlenbeck and Leonard S Ornstein. On the the-591 ory of the brownian motion. Physical Review, 36(5):823,592 1930.

[2] Evelyn F. Keller and Lee A. Segel. Initiation of slimes94 mold aggregation viewed as an instability. Journal of 595 Theoretical Biology, 26(3):399-415, 1970.

[3] L. Liang Li, S. F. Nrrelykke, and E. C. Cox. Persistent597 cell motion in the absence of external signals: a search 598 strategy for eukaryotic cells. PLoS ONE, 3(5):e2093,599 2008.

[4] Peter Dieterich, Rainer Klages, Roland Preuss, and Al-601 brecht Schwab. Anomalous dynamics of cell migration.602 Proceedings of the National Academy of Sciences of the603 United States of America, 105(2):459-63, 2008.

5] Arpita Upadhyaya, Jean-Paul Rieu, James A Glazier, ${ }_{005}$ and Yasuji Sawada. Anomalous diffusion and non-606 Gaussian velocity distribution of Hydra cells in cellular607 aggregates. Physica A, 293(3-4):549-558, 2001.

[6] A Szabó, R Ünnep, E Méhes, W O Twal, W S Argraves,609 Y Cao, and A Czirók. Collective cell motion in endothe-610 lial monolayers. Physical Biology, 7(4):046007, 2010. ${ }_{611}$

[7] András Czirók, Katalin Varga, Elod Méhes, and András612 Szabó. Collective cell streams in epithelial monolay-613 ers depend on cell adhesion. New Journal of Physics, 614 15:075006, 2013.

[8] C. L. Stokes, D. A. Lauffenburger, and S. K. Williams. 616 Migration of individual microvessel endothelial cells:617 Stochastic model and parameter measurement. Journal618 of Cell Science, 99:419-430, 1991.

[9] G. A. Dunn and A. F. Brown. A unified approach to620 analysing cell motility. Journal of Cell Science, 8:81-621 $192,1987$.

[10] Reinhold Fürth. Die brownsche bewegung bei623 berücksichtigung einer persistenz der bewegungsrichtung. 624 mit anwendungen auf die bewegung lebender infusorien.625 Zeitschrift für Physik A Hadrons and Nuclei, 2(3):244-626 256, 1920

11] H Risken. The Fokker-Planck Equation: Method of Solu-628 tion and Applications. Springer-Verlag, New York, 1989.629

[12] Hiroaki Takagi, Masayuki J. Sato, Toshio Yanagida, and630 Masahiro Ueda. Functional analysis of spontaneous cell631 movement under different physiological conditions. PLoS632 ONE, 3(7):e2648, 2008.

[13] D Selmeczi, S Mosler, PH Hagedorn, NB Larsen, and634 H Flyvbjerg. Cell motility as persistent random motion:635 theories from experiments. Biophysical Journal, 89:912-
931, 2005.

[14] L. Diambra, L. C. Cintra, Q. Chen, D. Schubert, and L. da F. Costa. Cell adhesion protein decreases cell motion: statistical characterization of locomotion activity. Physica A, 365:481-490, 2006.

[15] Nara Guisoni, Karina I. Mazzitello, and Luis Diambra. Modeling active cell movement with the Potts model. Frontiers in Physics, 6:61, 2018.

[16] Daniel Campos, Vicenç Méndez, and Isaac Llopis. Persistent random motion: uncovering cell migration dynamics. Journal of Theoretical Biology, 267:526-534, 2010.

[17] Fernando Peruani and Luis Morelli. Self-propelled particles with fluctuating speed and direction of motion in two dimensions. Physical Review Letters, 99:010602, 2007.

[18] Concepcion Tojo and Panos Argyrakis. Correlated random walk in continuous space. Physical Review E, 54:5863,1996

[19] Nicholas J. Savill and Paulien Hogeweg. Modelling morphogenesis: from single cells to crawling slugs. Journal of Theoretical Biology, 184(3):229-235, 1997.

[20] Joost B Beltman, Athanasius F M Marée, Jennifer N Lynch, Mark J Miller, and Rob J de Boer. Lymph node topology dictates $\mathrm{T}$ cell migration behavior. The Journal of Experimental Medicine, 204(4):771-80, 2007.

[21] Alexandre J. Kabla. Collective cell migration: leadership, invasion and segregation. Journal of The Royal Society, Interface, 9(77):3268-3278, 2012.

[22] L Bruno, V Levi, M Brunstein, and MA Despósito. Transition to superdiffusive behavior in intracellular actinbased transport mediated by molecular motors. Physical Review E, 80:011912, 2009.

[23] S. Bustingorry, E. R. Reyes, and Manuel O. Cáceres. Biased diffusion in anisotropic disordered systems. Physical Review E, 62:7664-7669, 2000.

[24] J P Rieu, A Upadhyaya, J Glazier, N B Ouchi, and Y Sawada. Diffusion and deformations of single hydra cells in cellular aggregates. Biophysical Journal, 79(4):1903-14, 2000.

[25] MA Despsito, C Pallavicini, V Levi, and L Bruno. Active transport in complex media: Relationship between persistence and superdiffusion. Physica A, 390:1026-1032, 2011.

[26] Carine P. Beatrici and Leonardo G. Brunnet. Cell sorting based on motility differences. Physical Review E, 84(3):031927, 2011. 
bioRxiv preprint doi: https://doi.orq/10.1101/2019.12.30.891093; this version posted December 30. 2019. The copyriaht holder for this preprint (which was not certified by peer review) is the author/funder, who has granted bioRxiv a license to display the preprint in perpetuity. It is made available under aCC-BY-NC-ND 4.0 International license.

[27] Sheng-You Huang, Xian-Wu Zou, and Zhun-Zhi Jin. Di-638 rected random walks in continuous space. Physical Re- view E, 65:052105, 2002. 